\title{
Image-Based Inertial Impact test for characterisation of strain rate dependency of Ti6Al4V titanium alloy.
}

\author{
T. Fourest · P. Bouda - L.C. Fletcher · D. Notta-Cuvier · \\ E. Markiewicz • F. Pierron · B. Langrand
}

Received: date / Accepted: date

T. Fourest

DMAS, ONERA, F-59014 Lille - France

Tel.: +33 320496900

Fax: +33 320496928

E-mail: thomas.fourest@onera.fr

P. Bouda

DMAS, ONERA, F-59014 Lille - France

E-mail: pascal.bouda@onera.fr

L.C. Fletcher

University of Southampton, Faculty of Engineering and Physical Sciences, Southampton - UK

E-mail: L.C.Fletcher@soton.ac.uk

D. Notta-Cuvier

Univ. Polytechnique Hauts-de-France, CNRS, UMR 8201 - LAMIH, Valenciennes F-59313, France

E-mail: Delphine.Notta@uphf.fr

E. Markiewicz

Univ. Polytechnique Hauts-de-France, CNRS, UMR 8201 - LAMIH, Valenciennes F-59313, France

E-mail: Eric.Markiewicz@uphf.fr

F. Pierron

University of Southampton, Faculty of Engineering and Physical Sciences, Southampton - UK

E-mail: F.Pierron@soton.ac.uk

B. Langrand

DMAS, ONERA, F-59014 Lille - France

E-mail: bertrand.langrand@onera.fr 
Abstract In the present work Image-Based Inertial Impact (IBII) tests are performed on Ti6Al4V material. The IBII test uses an impact on the edge of the specimen to generate a short pulse that loads the specimen. Three specimen geometries have been tested: a classic rectangular specimen, and two specimen geometries with stress concentrating geometries (i.e. a hole and notches) to enhance high levels of plastic strain. Full-field measurement of the acceleration and strain are successfully used in combination with the Virtual Fields Method (VFM) to identify the strain rate sensitivity parameter of the Johnson-Cook model. The strain/strain rate spectra covered by each specimen are analysed. Finally, the influence of the virtual field used in the identification process is discussed as well as the simultaneous identification of the Johnson-Cook model strain rate sensitivity parameter and the strain rate threshold parameter.

Keywords Inertial impact test · Titanium alloy · Virtual fields method · Full-field measurement · Viscoplasticity

\section{Introduction}

Material dynamic behaviour characterisation is often performed through standard tests. Their setup (possibly their processing) is often based on standards which have been initially defined for quasi-static tests. For instance, the standard ASTM E8-04 [1] reports a methodology for uniaxial tension testing of metallic materials. It relies on a statically determinate approach implying strong hypotheses on experimental test conditions. Thus, the analysis assumes a homogeneous level of strain and strain rate over the specimen gauge length. Assuming that the longitudinal nominal stress is proportional to the load measured from the test machine load cell, material parameters can be extracted from the uniaxial stress-strain curve. However, the extension of such standards to high strain-rate testing is not straightforward because of the difficulty achieving homogeneous mechanical fields, in particular for the strain rate [2]. For rate-dependent materials, the test campaign itself is more challenging because a high number of experiments is necessary with these approaches to characterise the behaviour over large spectra of strain and strain rate.

Owing to the dramatic improvements in imaging technology, it is now possible to measure kinematic fields at a large number of points over the surface of a test specimen (often referred to as "full-field measurements"). This is now also possible at high frame rates thanks to the growing technological developments in high speed cameras. Several inverse methods have been developed for identification of material parameters from full-field measurements. An overview of these methods is beyond the scope of this paper, but detailed reviews can be found in the literature, including for high strain-rate applications [2-4]. Among the available inverse methods, the Virtual Fields Method (VFM) was recently found to be of equivalent precision compared to the most widespread inverse method based on Finite Element simulations. However VFM was found to be 125 times faster in [5], and respectively 428 and 263 times faster for two cases in [6]. VFM can be used to identify material constitutive parameters from measurement of strain 
fields and either external forces [7] or acceleration fields [8]. The first approach (measuring external forces) was used to characterise viscoplastic Johnson-Cook (JC) model for titanium [9] and copper [10] alloys at moderately high strain rates (up to about $100 \mathrm{~s}^{-1}$ ) because it was not necessary to take inertial effects into account. The available literature about VFM with inertia effects for viscoplasticity characterisation $[11,12]$ extends to a study based on a bending test [13]. However, the specimen underwent small deformations only (0.02 at best) because the test configuration was specified without preliminary analyses. Therefore, the material behaviour was characterised on a limited viscoplastic spectra.

Image-Based Inertial Impact (IBII) tests have been recently proposed to take advantage of the VFM for material behaviour characterisation at high strain rate [14] (up to about $1000 \mathrm{~s}^{-1}$ ). It consists of a thin-plate specimen hit by a cylindrical projectile on its edge at several tens of meters per second, while the opposite edge is free to move. This leads to wave propagation / reflection travelling through the specimen and an additional longitudinal rigid body motion. Using this experimental setup, elastic [14] and elasto-plastic [15] constitutive models have been identified.

To extend the aforementioned tests to rate-dependent material behaviour, one needs to understand the influence of several tests parameters such as projectile velocity, specimen geometry and metrological features like camera specifications and full-field measurements regularisation parameters. Indeed, these parameters all influence the resulting identification and it is essential to set them using a rational approach. The development of such a methodology is presented in a companion paper by the authors [16]. The present work focuses on the application of the IBII test and VFM on Ti6Al4V samples to drastically reduce the test campaign needed for viscoplastic model identification on a wide viscoplastic spectra.

\section{Image-based Inertial Impact (IBII) test methodology}

The principle of IBII test has already been presented in the introduction section and discussed extensively in several papers $[14,15,17]$. So only the main concepts/tools used in the present work for its exploitation are briefly stated here, namely the VFM and grid method.

\subsection{The Virtual Fields Method (VFM)}

The Virtual Fields Method (VFM) [7] is based on the Principle of Virtual Work (PVW). For small perturbations, it can be expressed as follows:

$$
\begin{aligned}
-\int_{V} \underline{\underline{\sigma}}(\underline{X}): \underline{\underline{\varepsilon}}^{*} d V+\int_{\partial V} \underline{t} \cdot \underline{u}^{*} d S+\int_{V} \underline{f} \cdot \underline{u}^{*} d V & =\int_{V} \rho \underline{\gamma} \cdot \underline{u}^{*} d V \\
W_{\text {int }}^{*}(\underline{X})+W_{\text {ext }}^{*}+ & =W_{a c c}^{*}
\end{aligned}
$$


where $V$ denotes the material volume, $\underline{\underline{\sigma}}$ the Cauchy stress tensor computed from full-field strain measurements and constitutive laws using a set of material parameters $\underline{X} . \underline{u}^{*}$ is the virtual displacement field, $\underline{\underline{\varepsilon}}^{*}$ the virtual strain tensor such as $\underline{\underline{\varepsilon}}^{*}=\frac{1}{2}\left[\nabla \underline{u}^{*}+\left(\nabla \underline{u}^{*}\right)^{T}\right], \underline{t}$ the traction vector, $\underline{f}$ the body loads, $\rho$ the material density and $\underline{\gamma}$ the acceleration field. $W_{i n t}^{*}$ is the only term that depends on material parameters $\underline{X} . W_{e x t}^{*}$ is the external virtual work which comes from the contribution of the external loads during the test. $W_{a c c}^{*}$ is the contribution of acceleration which is also representative of inertia effects. As external loads are difficult to measure in transient dynamic tests, it is convenient to zero the virtual displacement field $\underline{u}^{*}$ at the loaded surfaces. In absence of body loads, the PVW is then expressed by:

$$
\begin{aligned}
\int_{V} \underline{\underline{\sigma}}[\underline{X}]: \underline{\underline{\varepsilon}}^{*} d V+\int_{V} \rho \underline{\gamma} \underline{\underline{u}}^{*} d V & =0 \\
-\quad W_{i n t}^{*}(\underline{X})+W_{a c c}^{*} & =0
\end{aligned}
$$

In the general case, the identification of non-linear material constitutive models with the VFM relies on the minimisation of a cost function, $\Phi$, describing a distance to equilibrium based on the PVW (Eqn. 1) [9, 18-20]. This cost function $\Phi$ may be defined by Eqn. 3, where $N_{i}$ is the number of monitored load steps (i.e. in practice the number of measured displacement maps).

$$
\min (\Phi[\underline{X}]) \text { with } \Phi[\underline{X}]=\sum_{i=1}^{N_{i}}\left[\frac{W_{a c c}^{*(i)}-W_{i n t}^{*(i)}[\underline{X}]}{W_{a c c}^{*(i)}}\right]^{2}
$$

The numerator denotes the gap to the PVW for a given time step (one image). The normalisation by $W_{a c c}^{*}$ ensures that all load steps can contribute equally to the cost function throughout the test. If only surface data are available from field measurement, uniform distribution through the thickness needs to be assumed. Here, the mechanical and kinematic fields will be assumed constant through the thickness using a plane stress assumption. So, the volume integral $V$ can be reduced to a surface integral $S$ over which mechanical fields are measured. In practice, this hypothesis is verified through numerical analysis based on finite element models of the test specimen. As full-field measurement techniques provide discrete data which are constant over the $k$-th elementary surface, the virtual field $\underline{u}^{*}$ and the related virtual strain tensor $\underline{\underline{\varepsilon}}^{*}$ can be averaged over each data point surface $S(k)$ (denoted $\bar{\varphi}^{S(k)}$ for any quantity $\varphi$ ). Then, Eqn. 3 can be written in discretized form as in Eqn. 4. Once the displacement maps are retrieved, numerical differentiation (single in space for $\underline{\underline{\varepsilon}}$ and double in time for $\underline{\gamma}$ ) enables the calculation of all virtual work components for a given set of material parameters $\underline{X}$.

$$
\Phi[\underline{X}]=\sum_{i=1}^{N_{i}}\left[1+\frac{\sum_{k=1}^{N} S(k)\left[\underline{\underline{\sigma}}[\underline{X}](i, k):{\underline{\underline{\varepsilon^{*}}}}^{N(k)}\right]}{\rho \sum_{k=1}^{N} S(k)\left[\underline{\gamma(i, k)} \cdot{\underline{\underline{u^{*}}}}^{S(k)}\right]}\right]^{2}
$$


The VFM has already been applied to characterise elastic [8,15,17,21-23], elastoplastic [15,24] and viscoplastic [11, 12] behaviour at high strain rate (up to $\propto 10^{3} s^{-1}$ ) using inertia as the load cell. In some cases, a Split Hopkinson Pressure Bar (SHPB) apparatus was used as the loading device $[8,12,21,24]$ though it was not well suited for the VFM. Indeed, the SHPB apparatus has been refined over the years to minimise inertia effects whereas here, we rely on these inertia effects to produce the load information. Thus, the novel IBII test was used $[14,15,17,23]$ to take full advantage of inertia effects in the VFM. The experimental setup consists of a cylindrical projectile propelled towards a thin plate specimen impacted in an edge-on configuration. The loading here is due to the propagation of the strain waves, generating high levels of acceleration which are beneficial to the VFM in dynamics.

Note that for IBII tests, $\underline{u}^{*}$ has to cancel the contribution of the impact force in the PVW (here at $x=0$, see Fig. 1). For instance, a 1D virtual field like in Eqn. 5 may be incorporated in Eqn. 4, where $L$ is the specimen length. As recommended in [8] the lowest order polynomial that satisfies the required constraints is used.

$$
\mid \begin{array}{l|l}
u_{x}^{*}=x / L & \varepsilon_{x x}^{*}=1 / L \\
u_{y}^{*}=0 & \varepsilon_{y y}^{*}=0 \\
\varepsilon_{x y}^{*}=0
\end{array}
$$

2.2 The grid method to extract strain and acceleration fields

Among available techniques for full-field measurements, the grid method has been recognised as a good compromise between spatial and displacement resolution, which favours accurate rendering of strongly heterogeneous kinematic fields when using low resolution cameras [25]. This is particularly relevant to the IBII method which relies on ultra-high speed cameras which tend to have low pixel counts. The grid method relies on imaging a periodic grid bonded onto the specimen surface to register its deformation between a reference and deformed states.

To extract phase maps (which carry the displacement information) from greyscale images, a spectrum analysis is employed [26]. Here, Local Spectrum Analysis (LSA) was performed using a Windowed Discrete Fourier Transform (WDFT). As is customary with grid method, the phase is defined modulo $2 \pi$. Thus, an unwrapping algorithm [27] was implemented to correct the stacking of phase maps from spatial and temporal phase jumps. Temporal jumps are caused by rigid body motion, and would strongly affect the calculation of the acceleration if ignored.

Displacement maps were then obtained with Eqn. 6, where $\underline{x}$ is the position vector, $\underline{u}$ the displacement vector, $\omega=\frac{2 \pi}{\Delta}$ the grid spatial frequency ( $\Delta$ the grid pitch), $\underline{\phi}^{R}$ and $\underline{\phi}^{D}$ reference and deformed phase maps respectively. Here, tested specimens undergo some rigid body motion owing to the nature of the test (impact with a free edge). 
Thus, the iterative approach described in [26] was used. This approach has the additional advantage that the grid pitch does not need to be completely regular as is the case with direct phase subtraction.

$$
\underline{u}[\underline{x}]=-\frac{1}{\omega}\left\{\underline{\phi}^{D}[\underline{x}+\underline{u}(\underline{x})]-\underline{\phi}^{R}[\underline{x}]\right\}
$$

As the WDFT analysis window spans two grid pitches, corrupted displacements are computed for the last grid pitch along the specimen edges. To replace them, Van Blitterswyk et al. [23] proposed to extrapolate data using local linear fitting. So far, this method has been used on straight edges only. Here, the method was extended to handle the case of curved edges (e.g. circular), as present in specimen geometries used in the present work. This method does not enforce the spatial continuity between the non-corrupted and reconstructed displacements.

Strain and acceleration maps (which are input into the VFM) are derived from displacement fields. As experimental displacement measurements contain noise, robust differentiators have been designed in the frequency domain [28]. They enable both low pass filtering of displacement fields and calculation of derivatives simultaneously. More precisely, a $n$-order differential of any quantity $\psi$ along $s$ is based on a centred kernel of neighbouring points $(i . e$. a weighted sum of discrete values of $\psi$ ). Finally $\left.\frac{\partial^{n} \psi}{\partial s^{n}}\right|_{m}$ is such as Eqn. 7, where $m$ is the step position, $N_{p}$ drives the kernel size, $\Delta s$ the pitch and $a_{i}(i \in \mathbb{N})$ are the weighting coefficients. Their expression is determined by enforcing high-order tangency of the robust differentiator magnitude response at high frequencies. More details can be found in [28]. In practice, these derivatives are computed through convolution of displacement fields $(\psi=\underline{u})$ maps with a sliding window based on coefficients $a_{i}$. Note that for $N_{p}=1$ the formula is equivalent to a central finite difference.

$$
\left.\frac{\partial^{n} \psi}{\partial s^{n}}\right|_{m}=\frac{1}{\Delta s^{n}}\left[a_{m} \psi_{m}+\sum_{i=1}^{N_{p}} a_{m-i} \psi_{m-i}-a_{m+i} \psi_{m+i}\right]
$$

\section{Experimental setup}

\subsection{Sample geometries}

Impact conditions must be set to enable viscoplasticity characterisation, i.e. to ensure the propagation of (visco)plastic wave in the specimens. As the studied material has a high initial yield stress (973 MPa [9]), it may be beneficial to "machine" stress concentrating geometries such as holes or notches to the rectangular specimen generally used in IBII tests. These geometries may help to ensure high levels of plastic strain and plastic strain rate. Three specimen geometries have been tested using the IBII configuration: a rectangular one (SMOOTH) as already used for elastic(plastic) behaviour characterisation in the literature $[14,15,17,23]$, a single-holed one (HOLE) and a double-notched one (NOTCH) with the notch location at the same x-coordinate (Fig. 1 with $x$ the impact direction). The latter has been chosen among hand-picked positions using preliminary Finite Element simulations to favour (visco)plastic wave 


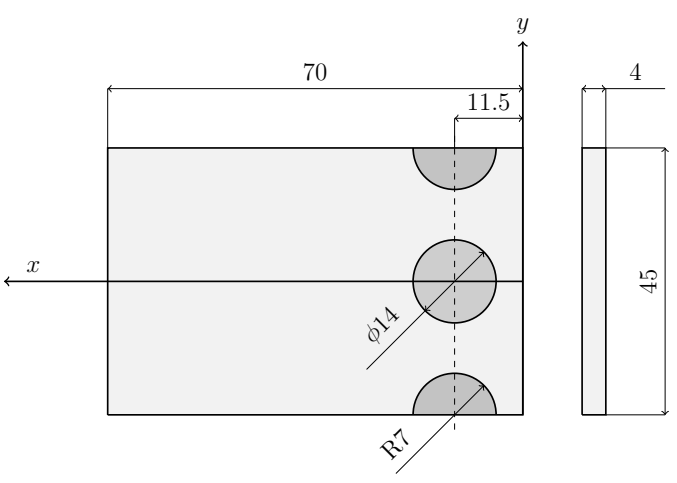

Fig. 1 Specimens geometries with the axis system used for the analysis of the experiments. SMOOTH (rectangular) - HOLE (single-holed) - NOTCH (double-notched). All dimensions in mm.

penetration. Note that the same amount of material has been "machined" so HOLE and NOTCH specimens have the same mass. As all specimens have the same length, the impact duration - that corresponds to the back-and-forth travelling time of a longitudinal elastic wave through the specimens - is always of about $25 \mu \mathrm{s}$, whatever the impact velocity.

\subsection{Impact test protocol}

The test campaign was performed at the University of Southampton using a custom impact rig designed to apply the IBII test methodology for material testing at high strain rate (Fig. 2). A breech system enables a cylindrical projectile to be accelerated towards the test specimen. The projectile is encased in a polymer sabot to reduce friction with the barrel. In the working section, specimens are glued to a waveguide with the same shape as the projectile. The waveguide lies on a 'V' shaped foam stand placed in front of the gas gun barrel before impact.

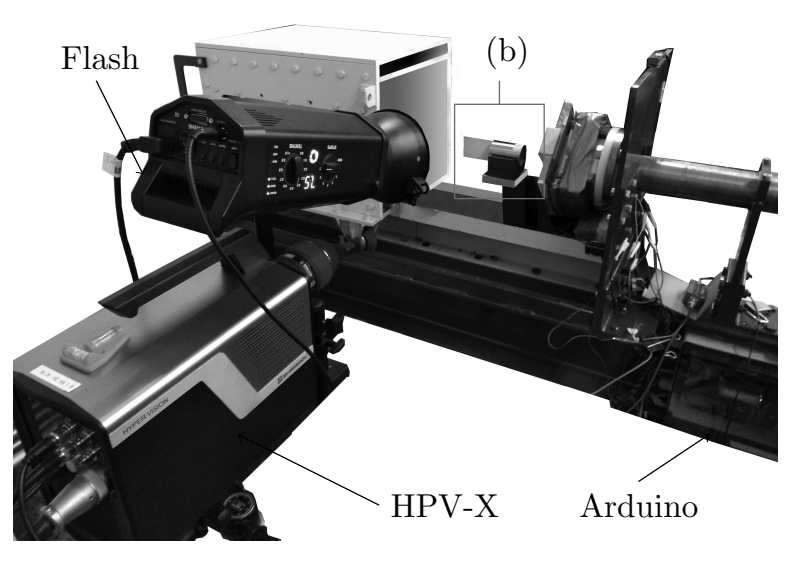

(a)

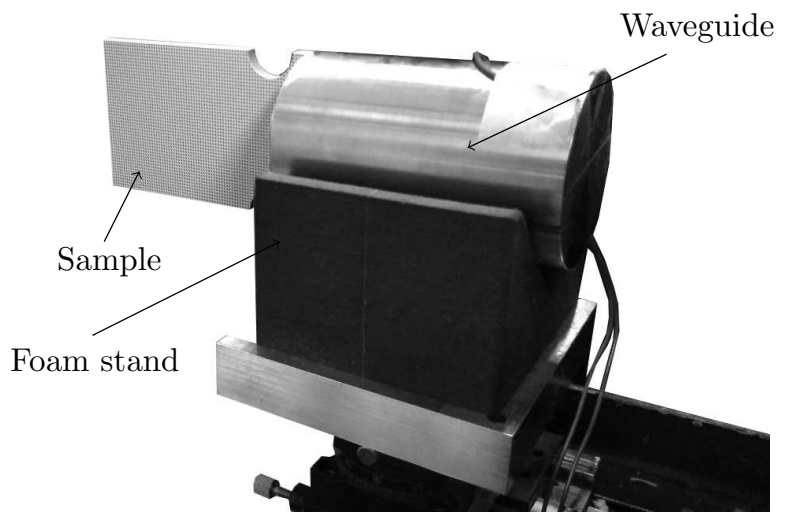

(b)

Fig. 2 Impact test setup main features - (a) imaging system - (b) waveguide and sample on foam stand 


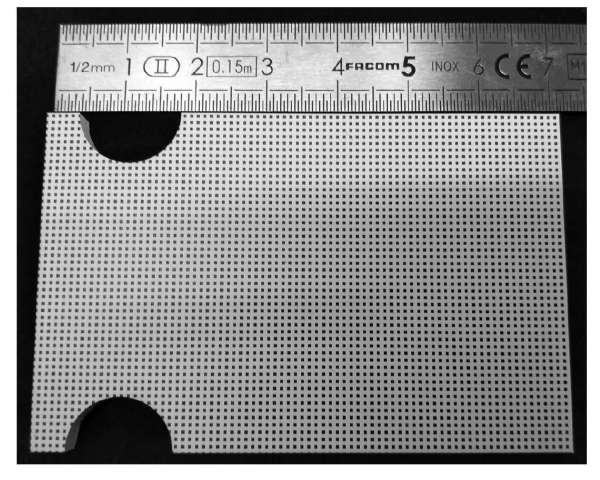

(a)

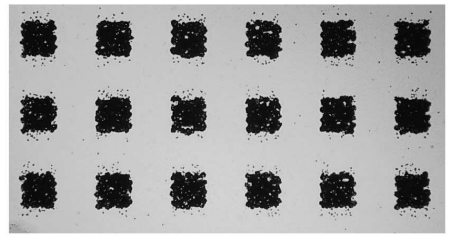

(b)

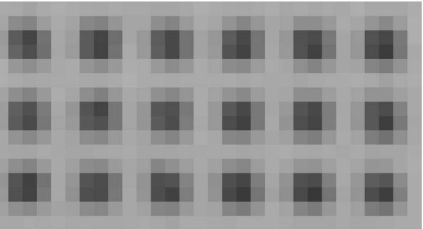

(c)

Fig. 3 Picture of a grid printed onto a notched specimen - (a) overall view - (b) x35 zoom - (c) HPV-X recording.

The imaging system consists of a Bowens Gemini GM100 Pro® flash and a Shimadzu HPV-X® camera. When the projectile hits the waveguide, an electrical circuit is closed triggering the ultra-high speed camera recording process. Prior to the impact, the projectile velocity is measured using light gates at the exit of the barrel and an Arduino Nano $囚$. The projectile speed and known range to the target is then used to calculate the specific delay required to trigger the flash accounting for its 100 microsecond rise time. This is crucial when using the HPV-X camera since "only" 128 frames can be recorded on the FTCMOS (chip-storage) sensors. To measure displacement fields, grids have been deposited onto the tested specimens. Black dots have been printed on a thin white paint coating with a Canon Océ Arizona $1260 \mathrm{XT} 囚$ printer [29,30]. Fig. 3 shows an example of these grids. Table 1 reports the main features of the metrological toolchain.

Table 1 Grid method and imaging parameters

\begin{tabular}{ll}
\hline Camera & Shimadzu HPV-X \\
Resolution & $400 \times 250$ pixels \\
Frame rate & $5 \mathrm{Mfps}$ \\
Sensor & FTCMOS (on-chip storage) \\
Dynamic range & 10 bits \\
Object pixel size & $0.182 \mathrm{~mm}$ \\
Grid pitch & 5 pixels $(0.91 \mathrm{~mm})$ \\
\hline
\end{tabular}

Projectiles and waveguides were made of maraging steel. The velocity was constrained by the maximal pressure level supplied (6 bars), corresponding to a projectile velocity of about $V_{p}=57 \mathrm{~m} \cdot \mathrm{s}^{-1}$. The geometry of the projectile and waveguide was the same with a length of $70 \mathrm{~mm}$ and a base radius of $24.5 \mathrm{~mm}$. For all performed tests the recorded impact velocity was between 55 and $58 \mathrm{~m} \cdot \mathrm{s}^{-1}$. Overall, eleven specimens with three different geometries were tested. 


\subsection{Metrological performance assessment}

Before material parameter identification using full-fields measurements, it is absolutely crucial to analyse the effect of regularisation on strain and acceleration resolution, especially for ultra-high speed imaging. Prior to each impact test, a set of 128 images was recorded for each specimen without loading (referred as the "static reference"). Processing these static image sequences with the grid method produced noise maps. From the noise maps the measurement resolution of each computed field was calculated as the standard deviation of each data set. Fig. 4 presents the typical noise error distribution for the longitudinal strain and longitudinal acceleration (note that longitudinal will hereafter be used to refer to components of the kinematic fields aligned with the 'x' axis shown in Fig. 1). Table 2 reports the resolutions obtained for strain and acceleration fields. They are computed with and without regularisation, i.e. $N_{p}=4$ and $N_{p}=1$ respectively in Eqn. 7 for both temporal and spatial differentiators. This value has been determined as optimal for spatial and temporal resolution for these tests in [31]. It is worth noting that only spatial regularisation is used to compute the strain fields and only temporal regularisation is applied to compute the acceleration field. The acceleration field is not computed for the raw data since noisy "static" fields lead to unrealistic estimations of the acceleration resolution, hence no resolution was reported here. When regularisation is used, the acceleration resolutions are in good accordance with the literature $[8,14,21]$. It was possible to analyse the raw strain resolution and, as expected, the strain resolution is increased with regularisation. Finally, it is worth noticing that the different specimens lead to approximatively the same resolutions.

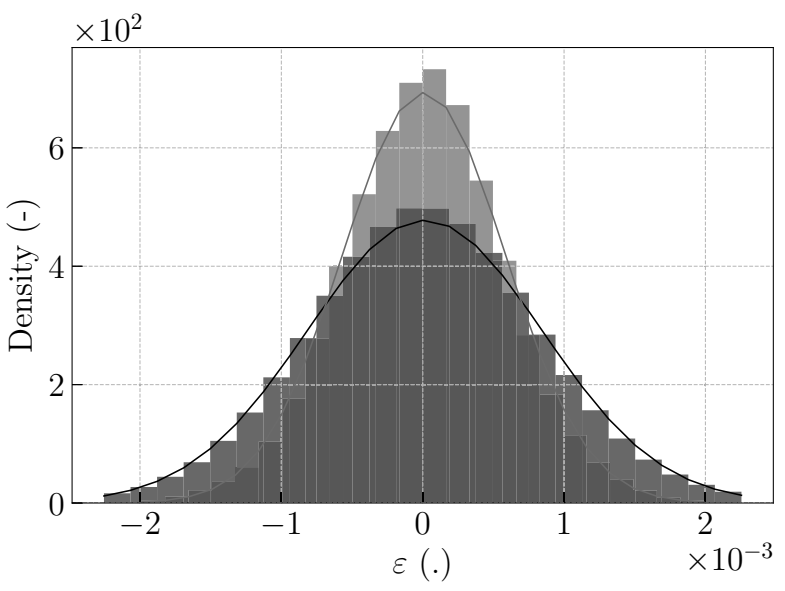

(a)

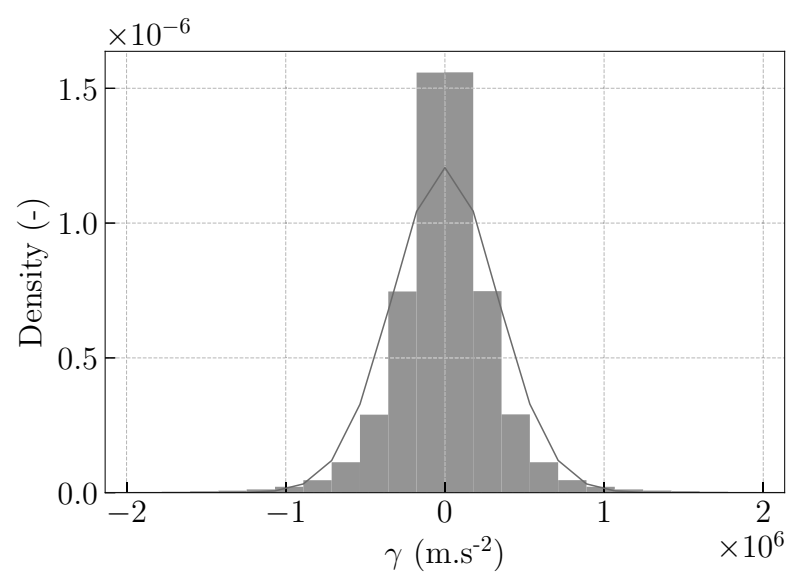

(b)

Fig. 4 Typical noise error distribution computed using the grid method in "static reference" tests (SMOOTH_01) - (a) longitudinal strain $\left(\varepsilon_{x x}\right)$ - (b) longitudinal acceleration $\left(\gamma_{x}\right)$ - Raw $(\square)$ - Regularised $(\square)$.

The effect of the temporal and spatial regularisation used for the computation of the longitudinal strain and longitudinal acceleration is illustrated in Fig. 5. Note that the spatial regularisation of the strain fields induces a 
Table 2 IBII tests measurement resolutions

\begin{tabular}{|c|c|c|c|c|}
\hline \multirow{2}{*}{ Test name } & \multicolumn{2}{|c|}{ Strain resolution $\left(\times 10^{-5}\right)$} & \multicolumn{2}{|c|}{ Acceleration resolution $\left(\times 10^{5} \mathrm{~m} \cdot \mathrm{s}^{-2}\right)$} \\
\hline & Raw & Regularised & Raw & Regularised \\
\hline SMOOTH-01 & 95 & 65 & - & 3.59 \\
\hline SMOOTH-02 & 100 & 64 & - & 3.40 \\
\hline NOTCH-01 & 136 & 53 & - & 3.12 \\
\hline NOTCH-02 & 95 & 63 & - & 3.49 \\
\hline NOTCH-03 & 141 & 78 & - & 4.28 \\
\hline NOTCH-04 & 123 & 90 & - & 3.59 \\
\hline NOTCH-05 & 200 & 68 & - & 3.33 \\
\hline HOLE-01 & 131 & 72 & - & 3.71 \\
\hline HOLE-02 & 206 & 90 & - & 3.64 \\
\hline HOLE-03 & 112 & 75 & - & 3.82 \\
\hline HOLE-04 & 121 & 68 & - & 3.5 \\
\hline
\end{tabular}

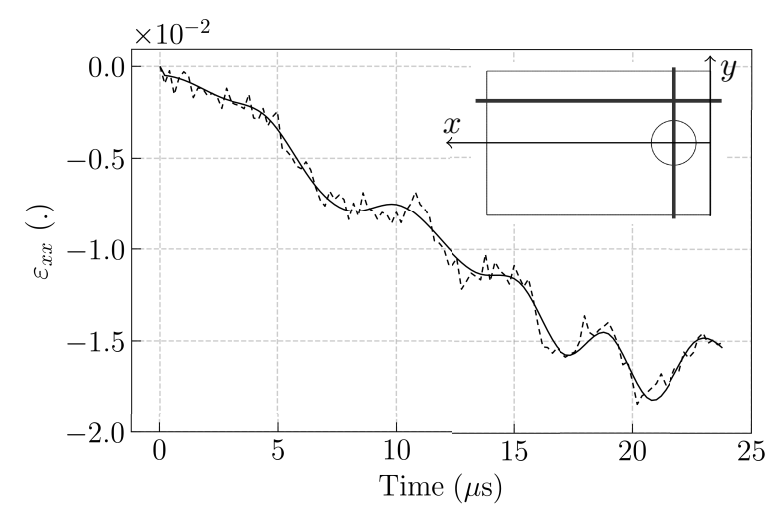

(a)

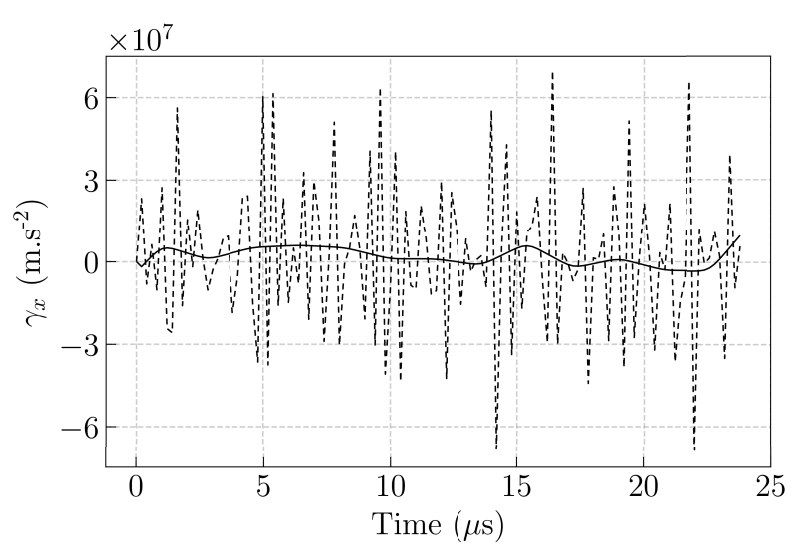

(b)

Fig. 5 Typical comparison between raw (- - ) and regularised (-) signals - (a) longitudinal strain $\left(\varepsilon_{x x}\right)$ - (b) longitudinal acceleration $\left(\gamma_{x}\right)$ for specimen HOLE-04 at position $\mathrm{x}=11.5 \mathrm{~mm}$ and $\mathrm{y}=13.1 \mathrm{~mm}$.

temporal smoothing. The temporal evolution of raw and regularised longitudinal strain are quite similar. However, as already explained the acceleration computed without temporal regularisation (double numerical derivation process) is very noisy (maximal amplitude over $5 \cdot 10^{7} \mathrm{~m} \cdot \mathrm{s}^{-2}$ as the maximal amplitude of the regularised one is $6 \cdot 10^{6} \mathrm{~m} . \mathrm{s}^{-2}$ ). The application of a regularisation process is very important because this quantity balances the internal work in the Principle of Virtual Work. 


\section{Experimental results}

4.1 Strain and acceleration extraction

Fig. 6 and Fig. 7 show typical maps of the longitudinal strain and acceleration for a sample of IBII tests at $t=t_{0}+20$ $\mu \mathrm{s}$, where $t_{0}$ is the time at which the compressive wave enters the specimen. This time corresponds approximately to $5 \mu \mathrm{s}$ before the end of a back-and-forth trip of the wave in the sample. Experimentally the longitudinal acceleration wave front took approximatively $13 \mu$ s to reach the free edge which is in good accordance with the analytical estimation $\Delta t=\frac{L_{s}}{\sqrt{E / \rho}} \approx 13 \mu \mathrm{s}$. Globally, one can observe that for all IBII tests, the wave propagation is not purely longitudinal (hence the break in the symmetry) because there was a slight misalignment between the waveguide and the projectile. However, this did not impede the test processing because it does not rely on the assumption that the test is purely uniaxial. One can notice that possible discontinuities due to displacement reconstruction in corrupted zones (i.e. at the specimen boundaries) does not seem to have large effects on the strain fields since strain computation involves a spatial kernel (Fig. 6). However discontinuities are observed in acceleration fields because its computation involve no spatial kernel (Fig. 7).

For the SMOOTH specimen, longitudinal strain levels reach about -0.01 at best whereas they reach more than -0.05 for the HOLE and NOTCH specimens (Fig. 6). Moreover, stress concentrating geometries enhanced the generation of strongly heterogeneous strain fields in their neighbourhood. However, these high levels of strain are localised to approximately one third of the specimen length. Therefore, the other part of the specimen undergoes mainly elastic strains. Accelerations are not influenced by the specimen geometry (Fig. 7): their peak levels are quite similar (about $10^{7} \mathrm{~m} . \mathrm{s}^{-2}$ ) and wave propagation is affected by misalignments.

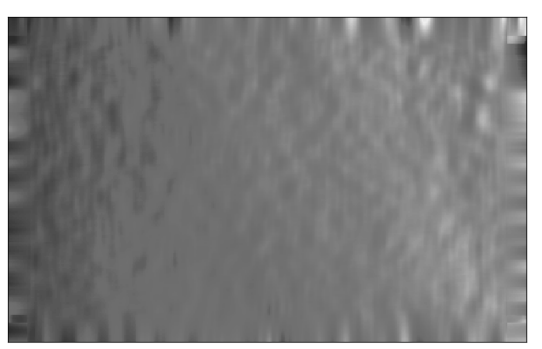

(a)

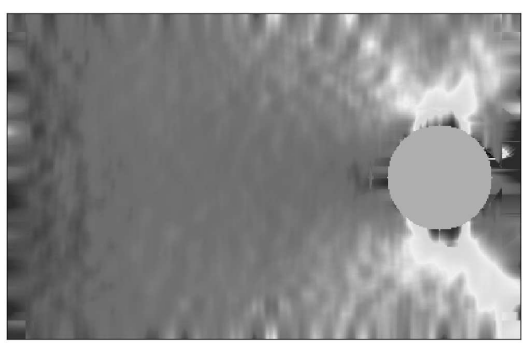

(b)

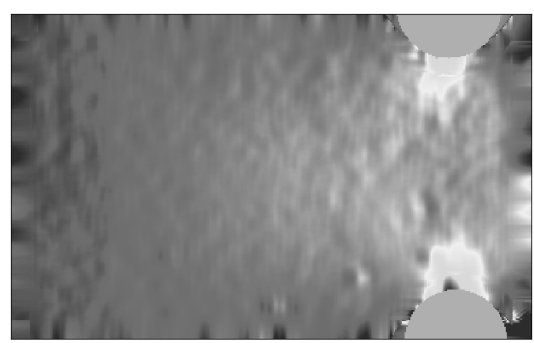

(c)

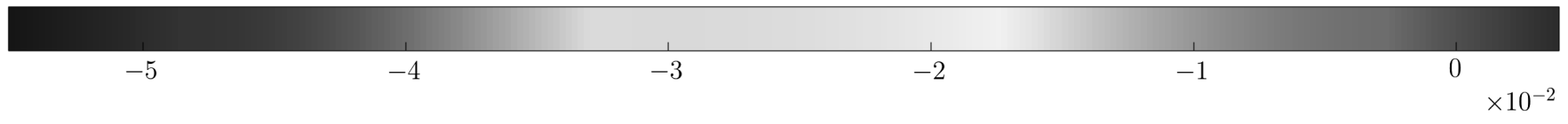

Fig. 6 Typical regularised longitudinal strain $\left(\varepsilon_{x x}\right)$ maps recorded for all specimen geometries at $t=t_{0}+20 \mu$ s, with $t_{0}$ the time corresponding to the entry of the compression wave in the specimen - (a) SMOOTH - (b) HOLE - (c) NOTCH. 


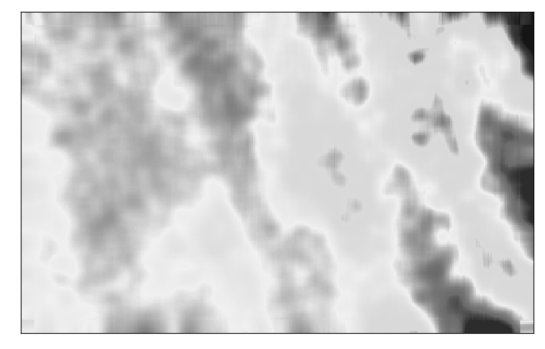

(a)

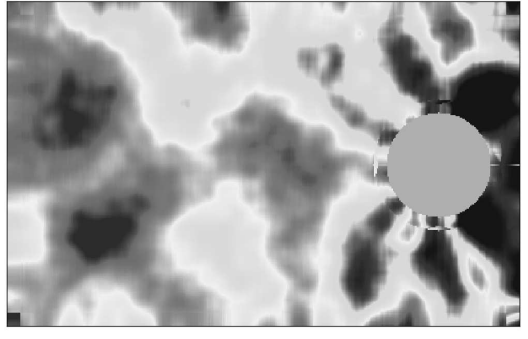

(b)

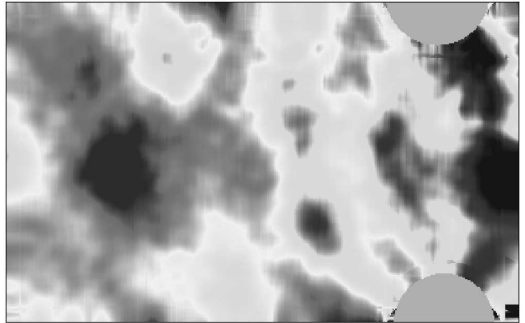

(c)

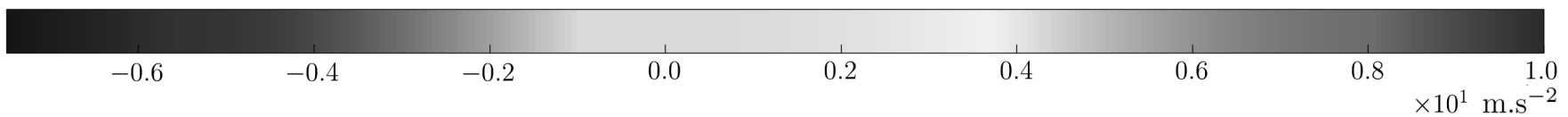

Fig. 7 Typical regularised longitudinal acceleration $\left(\gamma_{x}\right)$ maps recorded for all specimen geometries at $t=t_{0}+20 \mu \mathrm{s}$, with $t_{0}$ the time corresponding to the entry of the compression wave in the specimen - (a) SMOOTH - (b) HOLE - (c) NOTCH.

\subsection{Validation through dynamic VFM}

A way to assess the coherence of the measured strain and acceleration fields is to use the dynamic VFM (Eqn. 2) to calculate the Young's modulus. For the material under investigation the elastic modulus is not rate dependent so quasistatic test values will serve as reference value to compare to the value identified using the dynamic VFM. It consists of the reconstruction of the mean longitudinal stress $\overline{\sigma_{x x}}$ in a vertical slice of the specimen owing to acceleration fields (Eqn. 8) [14].

$$
\forall x, \overline{\sigma_{x x}}(x)=\frac{\rho}{D(x)} \sum_{j} \gamma_{x}(x, j) S(x, j)
$$

where $D(x)$ is the width of the specimen at abscissa $x$ and $j$ is the summation index over the area used for the reconstruction. Assuming an elastic material behaviour within a material slice, the Young's modulus $E$ can be identified using Eqn. 9. Finally, if Poisson's ratio $\nu=0.34$ [9] is known, stress/strain curves may be plotted for any slice to determine the $Q_{x x}$ stiffness, which is related to $E$ through Eqn. 9. Fig. 8 reports one example of stress/strain curve obtained at $\mathrm{x}=49.87 \mathrm{~mm}$ for a HOLE specimen.

$$
Q_{x x}=\frac{E}{1-\nu^{2}}=\frac{\overline{\sigma_{x x}}}{\overline{\varepsilon_{x x}+\nu \varepsilon_{y y}}}
$$

As expected the stress/strain curve is quite linear. Linearly fitting the stress/strain curve in Fig. 8 gives an elastic modulus of $\mathrm{E}=113 \mathrm{GPa}$, which is very close to the reference (value of the Young's modulus: 114 GPa [9]). For all tests, the identified elastic modulus was within $5 \%$ of the reference value. These results demonstrate the consistency of the strain and acceleration field computation. 


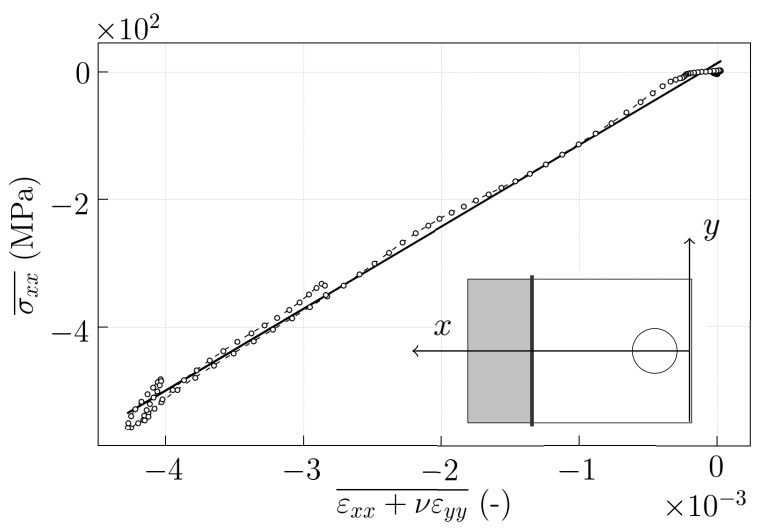

Fig. 8 Stress/strain curve for dynamic VFM (HOLE-04 sample and $\mathrm{x}=49.87 \mathrm{~mm})$ - Dynamic VFM (o) Linear fitting - $Q_{x x}=128$ GPa $(-)$. Vertical slice over which the mean stress is reconstructed $(-)$ - area embedding acceleration information ( $\square)$.

\section{Application of the dynamic VFM to viscoplasticity identification}

Now that strain and acceleration fields maps have been thoroughly assessed for the conducted IBII tests, they are used to identify parameters of the Johnson-Cook (JC) viscoplastic model with the dynamic VFM. The virtual field is given by Eqn. 5, and the cost function to be minimised in Eqn. 4. The JC model is introduced in the following section.

5.1 Johnson-Cook model for rate-dependent elasto-plastic material behaviour

Many constitutive models exists in the literature to describe the mechanical behaviour of metals, from early stages of loading until failure. For isotropic behaviour, constitutive models are generally based on von Mises (i.e. $J_{2}$-plasticity) theory, in conjunction with a hardening law which governs the evolution of the yield stress $\sigma_{y}[32]$ during loading. In the absence of kinematic hardening and damage, $\sigma_{y}$ is generally driven by accumulated plastic strain, $p$, or rate $\dot{p}$ and by temperature $T$. For rate-dependent behaviour characterisation, some of the existing models have a physical background (e.g. Zerilli-Amstrong [33] or Rusinek-Klepazcko [34]), such as dislocation mechanics and thermodynamics. However, they often involve a significant number of material parameters, hence are challenging to identify. By contrast, several phenomenological models (such as Cowper-Symonds [35], Johnson-Cook [36] or Jones [37]) have been developed as a compromise between computing efficiency and representation of physical phenomena. The Johnson-Cook (JC) model is the most used in finite element analysis. Its semi-empirical flow rule (Eqn. 10) can be split into three parts: a "static" term, $\sigma^{\text {stat }}$, similar to the Ludwik rate-independent isotropic hardening [32]; a "dynamic" correction term, $\sigma^{\text {visc }}$, activated if the equivalent strain-rate $\dot{p}$ reaches a threshold value $\dot{\varepsilon}_{e q, 0}$, and a third term, for thermal softening (not considered here see [36] for the full expression of the model). 


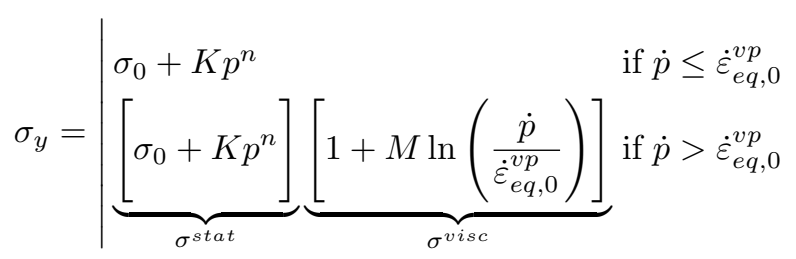

The JC model has been used to describe the behaviour of various metals such as iron [36, 38], copper [36, 38], steels [36, 38-41] or lightweight alloys [42-44]. In practice, if neglecting thermal softening, the identification of JC constitutive parameters includes two steps which rely on statically determinate approaches. First, quasi-static tests are performed to identify the material parameters driving $\sigma^{\text {stat }}$. Then, dynamic tests are performed to extract the parameters driving $\sigma^{\text {visc }}$ using a high-speed hydraulic machine and/or SHPB apparatus [45, 46] for instance. Finally, the combination of tests at low, moderate and high strain rate enables the characterisation of material behaviour over a large range of strain rates. However, numerous tests are necessary because of the assumption of constant and homogeneous strain rate fields for each specimen. In the present work the identification process is focused on the identification of the JC model viscoplastic parameter $M$.

\subsection{Strain rate sensitivity identification}

The Nelder-Mead algorithm was used to minimise the VFM cost function (Eqn. 4) in order to identify the value of the parameter $M$ using the eleven conducted IBII tests. So $\underline{X}$ in Eqn. 4 is reduced to the parameter $M$. Other parameters are fixed to their reference values, previously identified thanks to a statically determines approach [9] (Table 3). The stress in Eqn. 4 is computed using the measured strain field and a return mapping algorithm. For all identifications two initial guesses (two times and three times the reference value) have been used to verify that the minimisation process reaches the same final parameter value, which was always the case. In all performed identifications, the process reached convergence in less than 50 iterations (less than 10 minutes on a computer with an Intel@ i5 processor and 8 GB of RAM). The identification results are shown in Fig. 9 - (a). The JC viscoplastic parameter reference value $(M=0.033)$ obtained with standard tests [9] is also reported. In the present work, all identified values of parameter $M$ are in the range $[0.029,0.043]$. As there were enough tests, standard deviation and mean value were computed for the NOTCH and HOLE specimens. One can observe that in all cases, mean value $(M=0.035)$ is consistent with the reference value even if the latter has been identified for strain rate $\dot{\varepsilon}$ up to $500 \mathrm{~s}^{-1}$. This small difference might be explained by the fact that for the high plastic strain rate achieved in this study, the viscoplastic correction may not be perfectly linear with the logarithm of the plastic strain rate as it is assumed in the JC model (for instance an exponential function as in [38]). Nevertheless, one can note that in all cases, the reference value is well within the standard deviation range. It is also interesting that the standard deviation is smaller in the case of the HOLE specimen. 
Table 3 Ti6Al4V material constants for the Johnson-Cook's model [9]

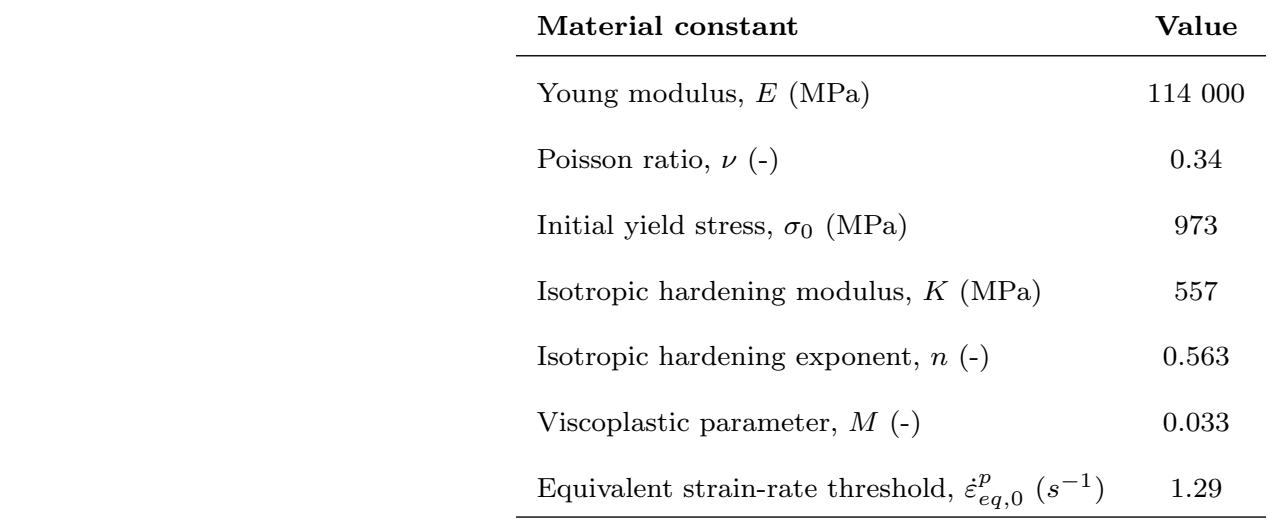

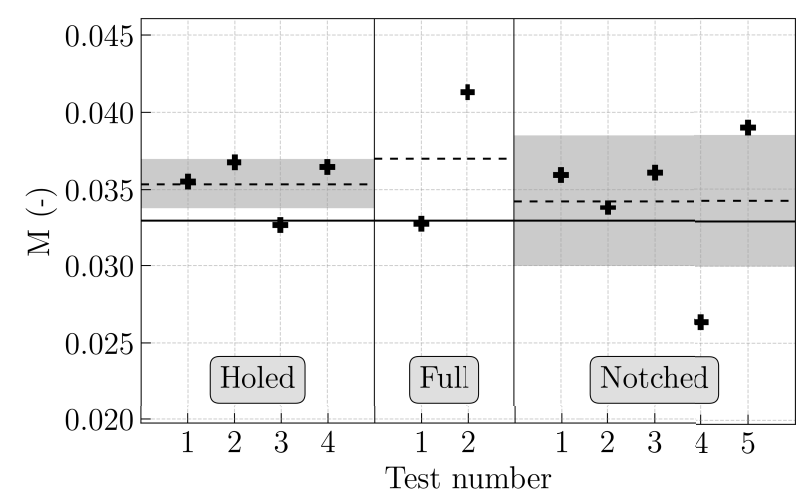

(a)

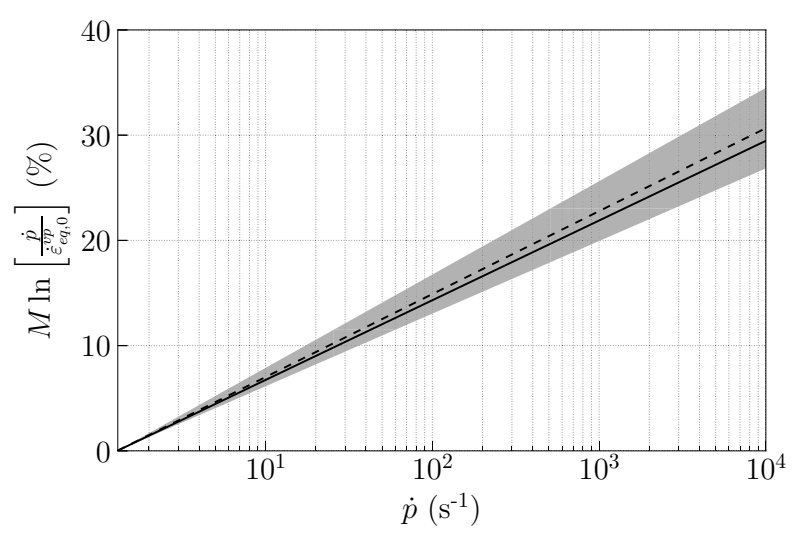

(b)

Fig. 9 JC viscoplastic parameter identified - (a) values for all performed tests - (b) Influence of M on yield stress multiplicative correction for the identification using HOLE samples. Reference (-) - VFM - Absolute (+) - VFM - Mean (- - -) - VFM - Standard deviation ( $\square$ ).

A way to analyse the influence of standard deviation on stress evaluation is to quantify the viscoplastic correction in the JC model for the identified values. Fig. 9 - (b) reports the evolution of the viscoplastic correction for these identified values on a wide range of plastic strain rate. One can observe that if $\dot{p}<10^{2} \mathrm{~s}^{-1}$ (intermediate rates), yield stress correction uncertainty is lower than 5\%. For higher values of $\dot{p}$, the uncertainty is higher. For NOTCH specimens, if $\dot{p}=10^{4} \mathrm{~s}^{-1}$ for instance, the uncertainty reaches $8 \%$ while the yield stress correction is about $35 \%$. So the effect of uncertainties on the yield stress proves to be quite reasonable especially for the high level of strain rate reached.

5.3 Viscoplastic spectra analysis

Fig. 10 shows the plastic strain distribution at the last recorded image of each specimen. It is computed using the identified value of $M$. As expected, the configuration without stress concentrating geometry provide the smallest range of plastic strains compared to those with notches and hole. Indeed, plastic strain levels of 0.01 are obtained at best with SMOOTH specimens. However, the NOTCH and HOLE specimens enable much higher levels of $p$ to be reached 
(about 0.08). It is clearly a first indicator of the advantage of using stress concentrating geometries in this type of test, especially for such a high yield stress material.

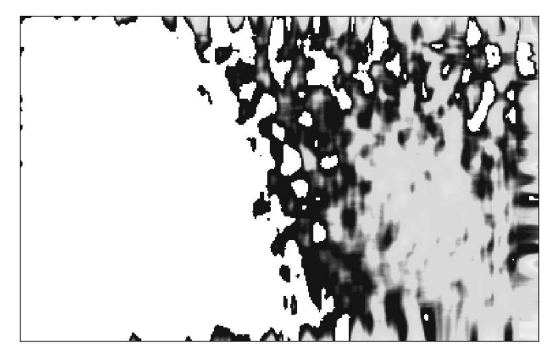

(a)

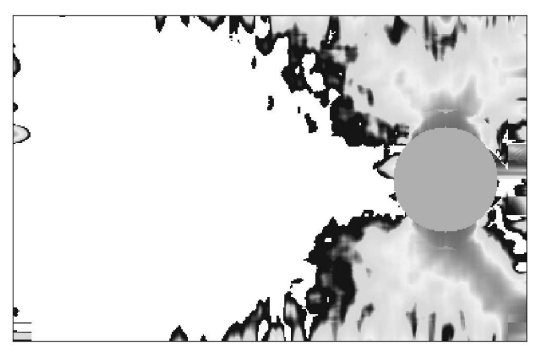

(b)

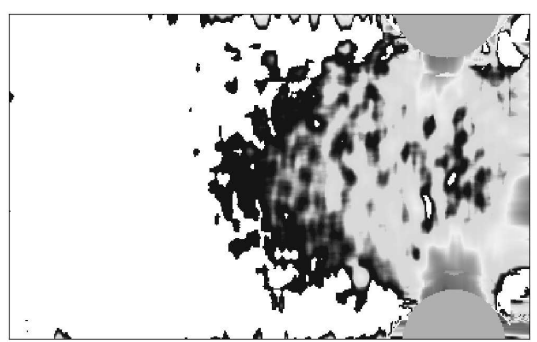

(c)

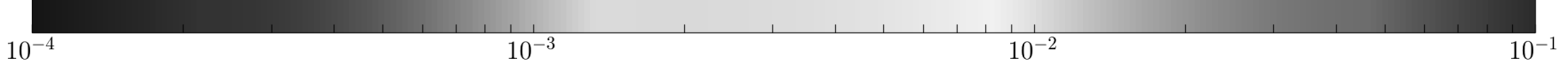

Fig. 10 Typical plastic strain $p$ maps recorded for all specimen geometry at $t=t_{0}+25 \mu$ s so for the last mechanical stage recorded with the high speed camera - (a) SMOOTH - (b) HOLE - (c) NOTCH. The parameter $M$ has been fixed to the mean of the identified values $M=0.035$, all other parameters are taken from [9].

Viscoplastic histograms were plotted for the conducted IBII tests, as shown in Fig. 11. All (visco)plastic states $(p, \dot{p})$ computed by the return mapping algorithm for the identified parameters are collected and counted in each test. A comparison between specimen geometries is reported in Fig. 11. One can observe that viscoplastic spectra are "band-shaped", i.e. high (visco)plastic strains occur at high strain rate and vice-versa. The spectra of the NOTCH and HOLE specimens overlap the spectrum of the SMOOTH specimen. However, there is an increase of approximately a decade in plastic strain and half a decade in plastic strain rate when comparing the HOLE and NOTCH geometry to the SMOOTH geometry. It is interesting that the HOLE and NOTCH geometries do not lead to the same spanning, even if the defects seem similar (same x position and same amount a material machined). Indeed, the HOLE specimen seems to cover a larger spectrum in the high plastic strain rate and high strain rate. The NOTCH specimen seems to cover a larger spectrum in the low plastic strain and plastic strain rate (around $10^{2} \mathrm{~s}^{-1}$ ).

\section{Discussion}

6.1 Virtual field sensitivity analysis

The dynamic VFM toolchain has been applied with a given methodology. This includes the virtual field defined by Eqn. 5 (referred as the linear virtual field) used in the computation of the PVW. This virtual field has been manually defined to be eligible for dynamic VFM, i.e. cancelling the contribution of external forces in the PVW. One way to assess the toolchain robustness is to analyse the influence of the choice of virtual field on the identification of $M$, 


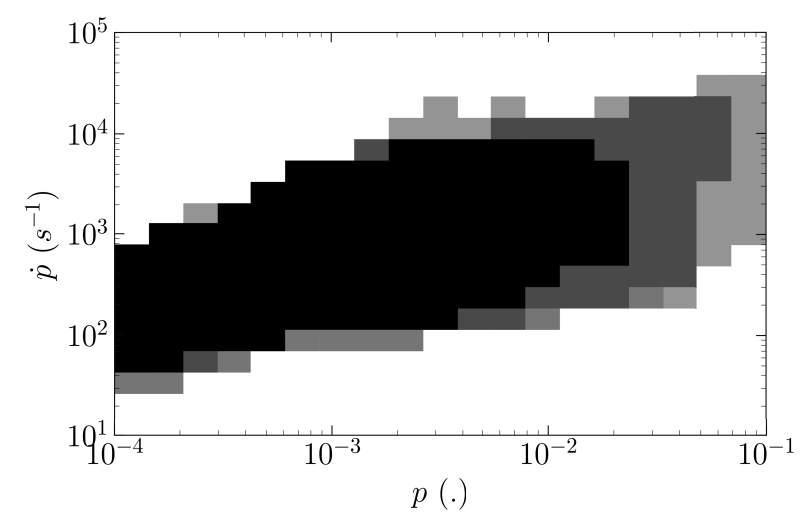

(a)

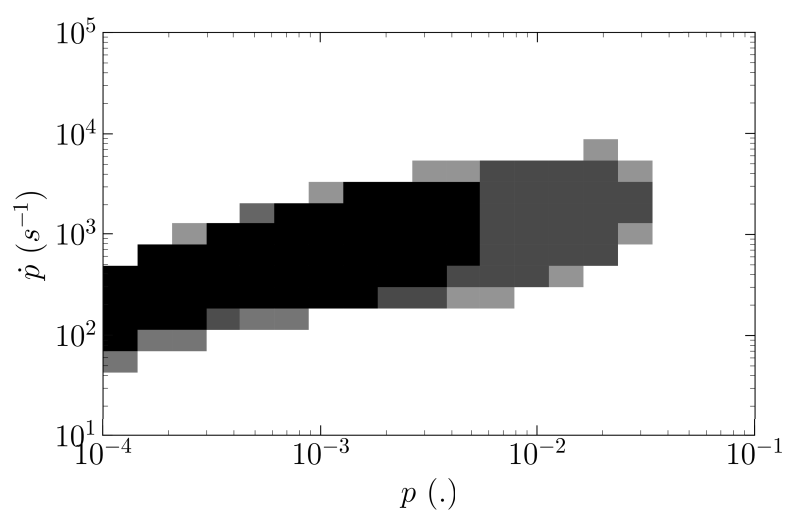

(b)

Fig. 11 Area of the $\dot{p} / p$ domain covered by each specimen geometry - (a) number of instance over 10 - (b) number of instance over $10^{3}$.

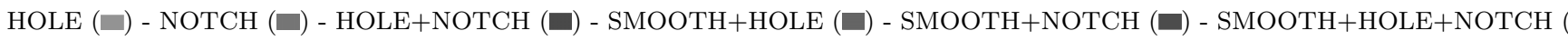

knowing that on noise-free data all virtual fields should give the same results. To do so, the previous identifications have been carried out with the virtual field defined by Eqn. 11 (referred as the quadratic virtual field). The results are compared to the one obtained with the linear virtual field in Fig. 12.

Each identification result is different when comparing the results of the linear and quadratic virtual fields. However, the results are in good accordance since the mean of the identified values are similar. One can observe that in the case of the HOLE specimens the use of the linear virtual field significantly decrease the standard deviation. This differences may be due to the biases owing to both data reconstruction and camera noise. Moreover, the virtual fields act like spatial filters. The linear field gives equal weight to all points on the strain field, unlike the quadratic one which weights arbitrarily the contribution of the specimen areas. So different result may be expected. As previously stated it is generally recommended to prefer low order virtual fields [8]. In fact, the definition of the virtual field is a key point of the identification process. Currently, the problem of selecting the best virtual fields in a general case is not solved yet [47].

$$
\mid \begin{array}{l|l}
u_{x}^{*}=x(x-L) & \varepsilon_{x x}^{*}=2 x-L \\
u_{y}^{*}=0 & \varepsilon_{y y}^{*}=0 \\
\varepsilon_{x y}^{*}=0
\end{array}
$$

\subsection{Multi-parameters identification}

In the present work, "only" the viscoplastic parameter $M$ of the JC model has been identified. Considering the spectra of data recorded during the tests (Fig. 11), it would seem legitimate to expect the simultaneous identification of both parameters of the dynamic correction part of the JC model, namely $M$ and $\dot{\varepsilon}_{e q, 0}^{v p}$. However, with the considered virtual fields in this paper (linear defined by Eqn. 5 and quadratic defined by Eqn. 11) and minimisation algorithm (Nelder- 


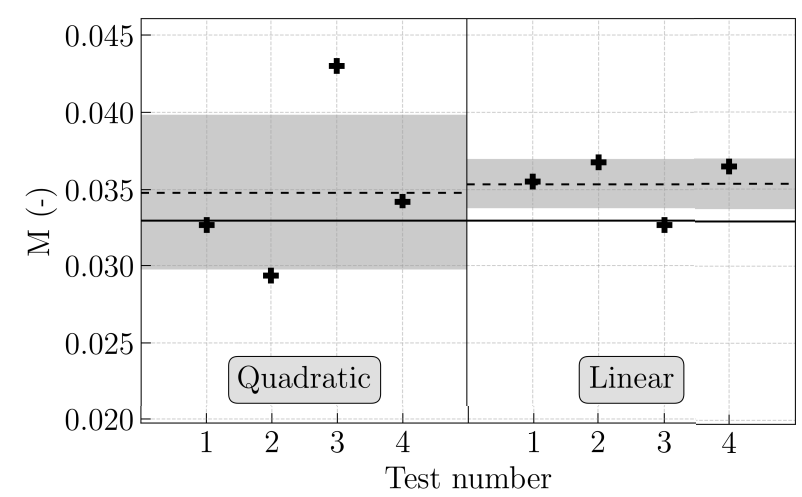

(a)

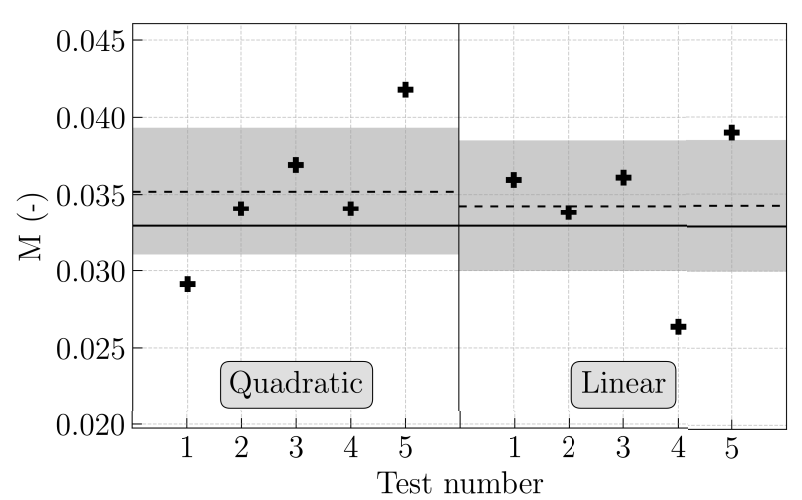

(b)

Fig. 12 Comparison of the identification performed using quadratic and linear virtual fields - (a) HOLE specimen - (b) NOTCH specimen. Reference (—), VFM - Absolute (+), VFM - Mean (- - -), VFM - Standard deviation ( $\square$ ).

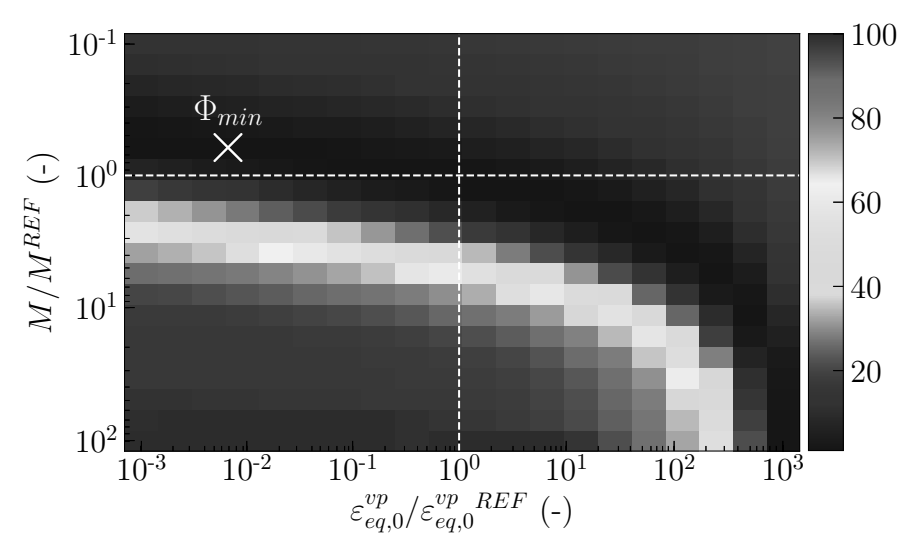

(a)

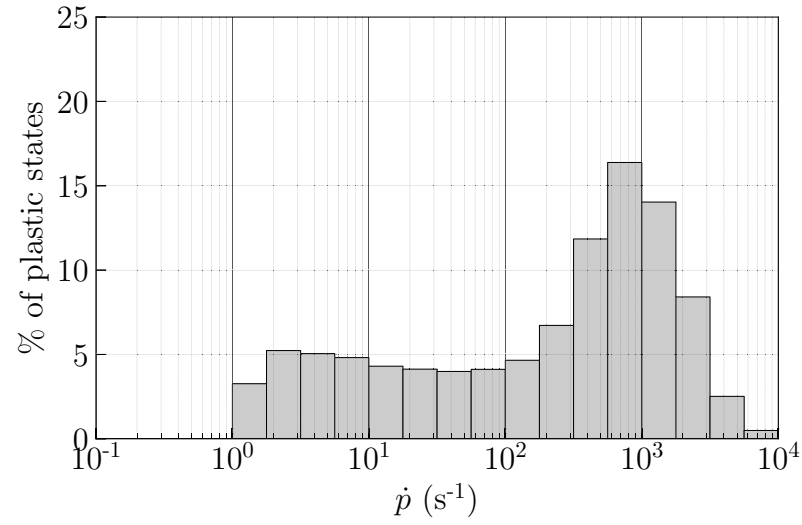

(b)

Fig. 13 (a) Cost function relative value $\Phi / \Phi_{\text {min }}: M$ vs $\dot{\varepsilon}_{e q, 0}^{v p}$ using the linear virtual field $\left(\Phi_{m i n}=1.63\right)$ - (b) viscoplastic histogram for the HOLE-04 test case.

Mead), the minimisation process leads to parameters values very different to the reference parameters (for instance for HOLE-4 specimen, $M=0.020, \dot{\varepsilon}_{e q, 0}^{v p}=6.87 \cdot 10^{-6} \mathrm{~s}^{-1}$ ) and very different between the considered test cases. This is also true using a more robust minimisation algorithm, such as the genetic CMAES [48].

Fig. 13 - (a) illustrates the dependency of the cost function to $M$ and $\dot{\varepsilon}_{e q, 0}^{v p}$ parameters. One can see that the cost function is convex but there is a large valley of low cost function values ranging from low to high values of both parameters. There are several local minima in this valley. Some parameters couples lead to unphysical results, for instance when the (multiplicative) correction overlaps the static hardening (for high values of $M$ ). Yet, those values are admissible from the dynamic VFM point of view since they enable the equilibrium between the $W_{i n t}^{*}$ and $W_{a c c}^{*}$ in the PVW. In the case of high values for $\dot{\varepsilon}_{e q, 0}$, the cost function imbalance is corrected owing to a large viscoplastic correction (high values of $M$ ) applied to a low number of plastic measurement points at the highest rates (Fig. 13 (b)). The opposite is also true: for low values for $\dot{\varepsilon}_{e q, 0}$, the cost function is balanced by a low viscoplastic correction applied to a large number of plastic measurement points. 
Different manually defined virtual fields were tested in order to try to change the shape of the cost function. However, in all the tested cases the valley already shown in Fig. 13 - (a) was still there. Recent developments have been made on the simultaneous identification of several parameters using VFM. In particular, virtual fields based on the sensitivity of the stress to the parameters variation have been investigated [49,50]. The definition of these sensitivity-based virtual fields is based on the detection of specimen area and time steps for which material behaviour exhibit a strong dependency to a given parameter (definition of one virtual field per parameter). Then, by giving increased weigth to these areas and time steps, the implication of the unknown parameter in the objective function is maximised, which facilitates its accurate identification but also reduces the influence of noise. It led to very interesting results on simulated data for the identification of anisotropic yield surfaces. Development of such a method for IBII tests and viscoplasticity identification seems very promising.

\section{Conclusions}

In the present work, Image-Based Inertial Impact tests have been performed on titanium alloy specimens. A specific difficulty in these tests is to apply a high amount of energy because the material has a high initial yield stress (973 $\mathrm{MPa}$ ). To ensure the penetration of the plastic wave in the specimen, two new specimen geometries involving stress concentrations, namely a hole and two notches have been introduced. Full-field measurements have been used to compute the strain and acceleration fields using spatial and temporal regularisation on displacements computed with the grid method. The consistency of measurements has been assessed by comparison of the strain and acceleration fields using the dynamic VFM to compute the elastic modulus of the material in areas away from the impact. It proved to be in very good agreement with the modulus identified using a statically determined approach [9].

Then, using these fields the strain rate dependency of the titanium alloy Ti6Al4V ( $M$ parameter of the JohnsonCook model) has been successfully identified. The identified parameter is very close to the reference values identified for strain rates up to $500 \mathrm{~s}^{-1}$ using a statically determined approach. The spectra of plastic strain/plastic strain rate covered in these IBII tests for all tested geometries have been compared. A gain of approximately one decade in plastic strain, and half a decade in plastic strain rate has been found for both the specimen geometries containing a stress concentration when compared to the standard rectangular geometry for the same impact velocity.

The main outlook of this work concerns the simultaneous identification of several parameters. In particular the identification of both parameters of the dynamic correction of the Johnson-Cook model seems achievable using the large spectra of data generated. Even if it is still challenging with the aforementioned VFM toolchain, several improvement 
paths may be explored such as improving the full-field measurement process (e.g. data reconstruction of corrupted areas) and the definition of the cost function (e.g. choice of the virtual fields [49]).

\section{Acknowledgments}

The authors are grateful to Onera and the Région Hauts-de-France for cofunding this project. Prof. Pierron acknowledges support by EPSRC through grant EP/L026910/1.

\section{References}

1. ASTM E8 - 04, Standard Test Methods for Tension Testing of Metallic Materials, ASTM International, West Conshohocken, PA, 2004.

2. E. Markiewicz, B. Langrand, and D. Notta-Cuvier. A review of characterisation and parameters identification of materials constitutive and damage models: From normalised direct approach to most advanced inverse problem resolution. International Journal of Impact Engineering, 110:371 - 381, 2017.

3. S. Avril, M. Bonnet, AS. Bretelle, M. Grédiac, F. Hild, P. Ienny, F. Latourte, D. Lemosse, S. Pagano, E. Pagnacco, and F. Pierron. Overview of identification methods of mechanical parameters based on full-field measurements. Experimental Mechanics, 48:381-402, 2008 .

4. M. Grédiac and F. Hild. Full-Field Measurements and Identification in Solid Mechanics. ISTE. Wiley, 2012.

5. L. Zhang, S.G. Thakku, M.R Beotra, M. Baskaran, T. Aung, J.C.H. Goh, N.G. Strouthidis, and M.J.A. Girard. Verification of a virtual fields method to extract the mechanical properties of human optic nerve head tissues in vivo. Biomechanics and modeling in mechanobiology, 16(3):871-887, 2017.

6. J.M.P. Martins, A. Andrade-Campos, and S. Thuillier. Comparison of inverse identification strategies for constitutive mechanical models using full-field measurements. International Journal of Mechanical Sciences, 145:330-345, 2018.

7. F. Pierron and M. Grédiac. The Virtual Fields Method: Extracting Constitutive Mechanical Parameters from Full-field Deformation Measurements. Springer New York, 2012.

8. R. Moulart, F. Pierron, S.R. Hallett, and M.R. Wisnom. Full-field strain measurement and identification of composites moduli at high strain rate with the virtual fields method. Experimental Mechanics, 51:509-536, 2011.

9. D. Notta-Cuvier, B. Langrand, E. Markiewicz, F. Lauro, and G. Portemont. Identification of Johnson-Cook's Viscoplastic Model Parameters Using the Virtual Fields Method : Application to Titanium Alloy Ti6Al4V. Strain, 49:22-45, 2013.

10. G. Valeri, B. Koohbor, A. Kidane, and M.A. Sutton. Determining the tensile response of materials at high temperature using DIC and the Virtual Fields Method. Optics and Lasers in Engineering, 91:53-61, 2017.

11. B. Koohbor, A. Kidane, M. A. Sutton, X. Zhao, and S. Mallon. Analysis of dynamic bending test using Ultra High Speed DIC and the virtual fields method. International Journal of Impact Engineering, 110:299 - 310, 2017. Special Issue in honor of Seventy Fifth Birthday of Professor N. K. Gupta.

12. B. Koohbor, A. Kidane, M. A. Sutton, X. Zhao, and S. Mallon. Corrigendum to "Analysis of dynamic bending test using Ultra High Speed DIC and the Virtual Fields Method" [Int. J. Impact Eng. 110 (2017) 299-310]. International Journal of Impact Engineering, 116:127 - 128, 2018. 
13. F. Pierron, M. A. Sutton, and V. Tiwari. Ultra High Speed DIC and virtual fields method analysis of a three point bending impact test on a aluminium bar. Experimental Mechanics, 51:537-563, 2010.

14. F. Pierron, H. Zhu, and C. Siviour. Beyond Hopkinson's bar. Philosophical Transactions of the Royal Society of London A: Mathematical, Physical and Engineering Sciences, 372, 2014.

15. S. Dreuilhe. Development of novel inertial tests for the identification of non-linear material model parameters at high strain rates. PhD thesis, University of Southampton, 2017.

16. P. Bouda, B. Langrand, D. Notta-Cuvier, E. Markiewicz, and F. Pierron. A computational approach to design new tests for viscoplasticity characterization at high strain-rates. Computational Mechanics, 2019.

17. L. Fletcher and F. Pierron. An Image-Based Inertial Impact (IBII) Test for Tungsten Carbide Cermets. Journal of Dynamic Behavior of Materials, Aug 2018.

18. S. Avril, F. Pierron, M. A. Sutton, and J. Yan. Identification of elasto-visco-plastic parameters and characterization of Lüders behavior using digital image correlation and the virtual fields method. Mechanics of Materials, 40:729-742, 2008.

19. F. Pierron, S.Avril, and V. T. Tran. Extension of the virtual fields method to elasto-plastic material identification with cyclic loads and kinematic hardening. International Journal of Solids and Structures, 47:2993-3010, 2010.

20. D. Notta-Cuvier, B. Langrand, F. Lauro, and E. Markiewicz. An innovative procedure for characterising a coupled elastoplastic damage model of behaviour using the virtual fields method. International Journal of Solids and Structures, 69-70:415-427, 2015.

21. F. Pierron and P. Forquin. Ultra-high-speed full-field deformation measurements on concrete spalling specimens and stiffness identification with the virtual fields method. Strain, 48:388-405, 2012.

22. S.-H. Yoon, M. Winters, and C.R. Siviour. High Strain-Rate Tensile Characterization of EPDM Rubber Using Non-equilibrium Loading and the Virtual Fields Method. Experimental Mechanics, 56:25-25, 2016.

23. J. Van Blitterswyk, L. Fletcher, and F. Pierron. Image-Based Inertial Impact Test for Composite Interlaminar Tensile Properties. Journal of Dynamic Behavior of Materials, Sep 2018. Available online.

24. G. Le Louëdec, F. Pierron, M. A. Sutton, C. Siviour, and A. P. Reynolds. Identification of the Dynamic Properties of Al5456 FSW Welds Using the Virtual Fields Method. Journal of Dynamic Behavior of Materials, 1(2):176-190, Jun 2015.

25. M. Grédiac, B. Blaysat, and F. Sur. A Critical Comparison of Some Metrological Parameters Characterizing Local Digital Image Correlation and Grid Method. Experimental Mechanics, 57(6):871-903, 2017.

26. M. Grédiac, F. Sur, and B. Blaysat. The Grid Method for In-plane Displacement and Strain Measurement: A Review and Analysis. Strain, 52(3):205-243, 2016.

27. H. Abdul-Rahman, M. Gdeisat, D. Burton, and M. Lalor. Fast three-dimensional phase-unwrapping algorithm based on sorting by reliability following a non-continuous path. In Proc. SPIE, volume 5856, pages 32-40, 2005.

28. P. Holoborodko. (n.d) Smooth noise-robust differentiators. Retrieved from http://www/holoborodko.com/pavel/numericalmethods/noise-robust-smoothing-filters/.

29. Y. Surrel. Grid printing part I. http://photodyn.org/wp-content/uploads/2016/03/Report_UniversityPrintCentre.pdf, 2018.

30. Y. Surrel. Grid printing part II. http://photodyn.org/wp-content/uploads/2016/03/Report_UniversityPrintCentre_2.pdf, 2018.

31. P. Bouda. Méthode des Champs Virtuels pour la caractérisation du comportement dynamique de matériaux métalliques sous chargement purement inertiel. PhD thesis, Université Polytechnique Hauts-de-France, 2019.

32. J. Lemaitre and J.L. Chaboche. Mechanics of Solid Materials. Cambridge University Press, 1994.

33. F. J. Zerilli and R. W. Armstrong. Dislocation mechanics based constitutive relations for material dynamics calculations. Journal of Applied Physics, 61(5):1816-1825, 1987. 
34. A. Rusinek and J.R Klepaczko. Shear testing of a sheet steel at wide range of strain rates and a constitutive relation with strain-rate and temperature dependence of the flow stress. International Journal of Plasticity, 17(1):87 - 115, 2001.

35. G.R. Cowper and P.S. Symonds. Strain-hardening and Strain-rate Effects in the Impact Loading of Cantilever Beams. Technical report (Brown University. Division of Applied Mathematics). Division of Applied Mathematics, Brown University, 1957.

36. G.R. Johnson and W.H. Cook. A constitutive model and data for metals subjected to large strains, high strain rates and high temperatures. Proc. 7th International Symposium on Balistics, The Hague, The Netherlands, pages 541-547, 1983.

37. Norman Jones. Structural Impact. Cambridge University Press, 1990.

38. T. J. Holmquist and G. R. Johnson. Determination of constants and comparison of results for various constitutive models. J. Phys. IV France, 01:C3-853-C3-860, 1991.

39. T. Borvik, M. Langseth, O.S. Hopperstad, and K.A. Malo. Ballistic penetration of steel plates. International Journal of Impact Engineering, 22:855-886, 1999.

40. A. K. Gupta, H. N. Krishnamurthy, P. Puranik, S. K. Singh, and A. Balu. An exponential strain dependent Rusinek-Klepaczko model for flow stress prediction in austenitic stainless steel 304 at elevated temperatures. Journal of Materials Research and Technology, $3(4): 370-377,2014$

41. M.A. Iqbal, K. Senthil, P. Sharma, and N.K. Gupta. An investigation of the constitutive behavior of Armox 500T steel and armor piercing incendiary projectile material. International Journal of Impact Engineering, 96:146 - 164, 2016.

42. H.W. Meyer Jr and D.S. Kleponis. Modeling the high strain rate behavior of titanium undergoing ballistic impact and penetration. International Journal of Impact Engineering, 26:509-521, 2001.

43. J. Peirs, P. Verleysen, W. Van Paepegem, and J. Degrieck. Determining the stress-strain behaviour at large strains from high strain rate tensile and shear experiments. International Journal of Impact Engineering, 38:406-415, 2011.

44. W. Mocko and A. Brodecki. Application of optical field analysis of tensile tests for calibration of the Rusinek-Klepaczko constitutive relation of Ti6Al4V titanium alloy. Materials and Design, 88:320-330, 2015.

45. B. Hopkinson. A method of measuring the pressure produced in the detonation of high explosives of by the impact of bullets. Philosophical Transactions of the Royal Society A, 213:437-456, 1914.

46. T. Nicholas. Tensile testing of materials at high rates of strain. Experimental Mechanics, 21(5):177-185, May 1981.

47. M. Grédiac, F. Pierron, S. Avril, and E. Toussaint. The Virtual Fields Method for Extracting Constitutive Parameters From Full-Field Measurements: a Review. Strain, 42:233-253, 2006.

48. N. Hansen and A. Ostermeier. Completely derandomized self-adaptation in evolution strategies. Evolutionary computation, 9(2):159$195,2001$.

49. A. Marek, F. M. Davis, and F. Pierron. Sensitivity-based virtual fields for the non-linear virtual fields method. Computational Mechanics, 60(3):409-431, Sep 2017.

50. A. Marek, F. Davis, M. Rossi, and F. Pierron. Extension of the sensitivity-based virtual fields to large deformation anisotropic plasticity. International Journal of Material Forming, 12(3):457-476, 2019. 